\title{
Nonparametric approximation of conditional risk in nonstationary geostatistical processes
}

\section{Rubén Fernández-Casal}

Universidade da Coruña: Universidade da Coruna

\section{Sergio Castillo-Páez}

ESPE: Universidad de las Fuerzas Armadas

Mario Francisco-Fernández ( $\triangle$ mariofr@udc.es)

Universidade da Coruña https://orcid.org/0000-0002-9201-5423

\section{Research Article}

Keywords: Bootstrap, Conditional Risk, Conditional simulation, Local linear estimation

Posted Date: March 8th, 2022

DOI: https://doi.org/10.21203/rs.3.rs-1398694/v1

License: (c) (i) This work is licensed under a Creative Commons Attribution 4.0 International License.

Read Full License 


\title{
Nonparametric approximation of conditional risk in nonstationary geostatistical processes
}

\author{
Rubén Fernández-Casal ${ }^{1 \dagger}$, Sergio Castillo-Páez ${ }^{2 \dagger}$ and Mario \\ Francisco-Fernández ${ }^{1 * \dagger}$
}

${ }^{1 *}$ Departamento de Matemáticas, Universidade da Coruña. CITIC, Facultad de Informática, Campus de Elviña s/n, A Coruña, 15071, Spain.

${ }^{2}$ Departamento de Ciencias Exactas, Universidad de las Fuerzas Armadas ESPE, Av. General Rumiñahui s/n, Sangolquí, 171103, Ecuador.

*Corresponding author(s). E-mail(s): mariofr@udc.es;

Contributing authors: ruben.fcasal@udc.es; sacastillo@espe.edu.ec;

$\dagger$ These authors contributed equally to this work.

\begin{abstract}
A nonparametric procedure to estimate the conditional probability that a geostatistical process exceeds a certain threshold value is proposed. The method consists of a bootstrap algorithm that combines conditional simulation techniques with nonparametric estimations of the trend and the variogram. The nonparametric local linear estimator, considering a bandwidth matrix selected by a method that takes the spatial dependence into account, is used to estimate the trend. The variogram is approximated by a flexible bias-corrected estimator based on the residuals. The proposed method allows to obtain estimates of the conditional exceedance risk in non-observed spatial locations. The performance of the approach is analyzed by simulation and illustrated with the application to a real data set.
\end{abstract}

Keywords: Bootstrap, Conditional Risk, Conditional simulation, Local linear estimation 
Nonparametric estimation of conditional risk

\section{Introduction}

Risk maps containing the probabilities that a certain variable of interest exceeds a given threshold or permissible value in an area of study are usually employed by environmental agencies to control different pollution levels (in soil, air or water) or to alert population of possible natural disasters (earthquakes, floods, etc.). The estimation of these exceeding probabilities using simple and reliable statistical methods is, therefore, an important practical issue. The resulting estimated maps can help governments to make decisions and to organize prevention policies in the near future.

Different geostatistical techniques have usually been employed to approximate exceeding probabilities. These methods include traditional approaches, such as the indicator kriging (IK) (e.g. Goovaerts et al, 1997) or the disjunctive kriging (DK) (e.g. Webster and Oliver, 1989), or more recent procedures, such us those based on analysis of compositional data (e.g. Tolosana-Delgado et al, 2008). The IK consists in the application of the simple kriging linear predictor to indicator functions of the data. Although it is perhaps the most popular approach in this context, it has some drawbacks. First, the discretization of the data can lead to a loss of information. On the other hand, the estimated probabilities could be greater than one or negative. Moreover, it could present order-relation problems (see, e.g. Chilès and Delfiner, 2012, Section 6.3.3). Some of these issues can be avoided with the use of the so-called simplicial indicator kriging (Tolosana-Delgado et al, 2008). This method employs a simplex approach for compositional data to estimate the conditional cumulative distribution function. Another alternative to the IK is the DK, a nonlinear estimation technique which usually assumes a Gaussian isofactorial model for the geostatistical process. However, there is no empirical evidence to recommend the DK in preference to the IK, or the opposite (Lark and Ferguson, 2004).

The approaches previously described usually suppose stationarity and a parametric model. Therefore, if the assumed model is not appropriate, the conclusions drawn may be unreliable or even wrong. To avoid these problems, alternatively, nonparametric techniques could be used. In this line, FernándezCasal et al (2018) proposed an unconditional bootstrap method to estimate the spatial risk, without assuming any parametric form for the trend function nor for the dependence structure of the process. They used local linear estimates of the trend and the variogram, jointly with a procedure to correct the bias introduced by the direct use of the residuals in the variogram estimation. Although this procedure avoids misspecification problems, it focuses on the estimation of the unconditional probability that the variable under study exceeds a threshold. Note that, as the replicas obtained by unconditional simulation will not necessarily match the observed sample values (see, e.g. Chilès and Delfiner, 2012, Chapter 7), a direct comparison of this procedure with the previous ones is not entirely appropriate, since they aim at the estimation of conditional exceeding probabilities.

In the present work, we propose a bootstrap method to estimate threshold exceeding conditional probabilities. This approach uses a modified version 
of the unconditional bootstrap method introduced in Fernández-Casal et al (2018) as part of its implementation. The new procedure generates condicional replicates matching up the observed values at the sampled locations. The conditionalization of simulations is equivalent to choose among all possible unconditional simulations of the spatial process, those that coincide with the values obtained at the observation locations (Journel, 1974).

The remainder of the paper is organized as follows. In Section 2, the spatial model considered in this research is presented. Additionally, the nonparametric estimators for the mean or trend function and for the variogram employed in the conditional bootstrap method are introduced. In Section 3, the bootstrap algorithm to estimate the conditional risk is described. In this procedure, a slight modification of the bootstrap method proposed in Fernández-Casal et al (2018) to approximate the unconditional risk (also dicussed in this section) is used. A simulation study for assessing the performance of the new approach, considering stationary and nonstationary processes, is provided in Section 4. An example of application of the methods to precipitation data is included in Section 5. Finally, Section 6 contains some conclusions and finals remarks.

\section{Geostatistical model and nonparametric estimators}

Given the spatial process $\left\{Y(\mathbf{x}): \mathbf{x} \in D \subset \mathbb{R}^{d}\right\}$, our interest focuses on the estimation of the conditional probability

$$
r_{c}\left(\mathbf{x}_{\alpha}^{e}, \mathbf{Y}\right)=P\left[Y\left(\mathbf{x}_{\alpha}^{e}\right) \geq c \mid \mathbf{Y}\right],
$$

where $\mathbf{Y}=\left[Y\left(\mathbf{x}_{1}\right), \ldots, Y\left(\mathbf{x}_{n}\right)\right]^{t}$ are observed values of the process at certain sample locations, $c$ is a threshold (critical) value, and $\left\{\mathbf{x}_{\alpha}^{e}\right\}_{\alpha=1}^{n_{0}}$ is a set of unobserved estimation locations.

We additionally assume that the spatial process is not stationary and it can be modeled as:

$$
Y(\mathbf{x})=\mu(\mathbf{x})+\varepsilon(\mathbf{x}),
$$

where $\mu(\cdot)$ is the trend function, and the error term $\varepsilon$ is a second order stationary process with zero mean and covariogram $C(\mathbf{u})=\operatorname{Cov}[\varepsilon(\mathbf{x}), \varepsilon(\mathbf{x}+\mathbf{u})]$, for $\mathbf{x}$ and $\mathbf{x}+\mathbf{u} \in D$.

The first step in the proposed approach consists in the nonparametric estimation of the trend and the dependence of the spatial process. For this, the same procedure to that proposed in Fernández-Casal et al (2018), briefly discussed in the following subsection, is used.

\subsection{Nonparametric trend and dependence estimation}

Assuming model (2) and from a nonparametric viewpoint, the main concern is usually the estimation of the trend $\mu(\cdot)$. Different nonparametric approaches have been used for this task, including kernel methods, splines or wavelets 
techniques. A comprehensive revision of some of these approaches can be found in Opsomer et al (2001). In that paper, the authors focus on the framework of regression models with correlated errors. Note that if the data are correlated, to obtain reliable results it is necessary to perform some adjustments in the approaches or in the methods employed to select the tuning parameters needed to apply the nonparametric techniques.

In the present paper, to estimate the trend $\mu(\cdot)$, we consider a kernel-based method called the local linear estimator. This approach has shown a very good performance from a theoretical and a practical point of view (e.g. Fan and Gijbels, 1996; Wand and Jones, 1995). In the spatial framework, given a sample $\left\{\left[\mathbf{x}_{i}, Y\left(\mathbf{x}_{i}\right)\right]\right\}_{i=1}^{n}$, the local linear estimator of $\mu(\mathbf{x})$ is given by:

$$
\hat{\mu}_{\mathbf{H}}(\mathbf{x})=\mathbf{e}_{1}^{t}\left(\mathbf{X}_{\mathbf{x}}^{t} \mathbf{W}_{\mathbf{x}} \mathbf{X}_{\mathbf{x}}\right)^{-1} \mathbf{X}_{\mathbf{x}}^{t} \mathbf{W}_{\mathbf{x}} \mathbf{Y} \equiv s_{\mathbf{x}}^{t} \mathbf{Y}
$$

where $\mathbf{e}_{1}$ is a vector with 1 in the first entry and all other entries $0, \mathbf{X}_{\mathbf{x}}$ is a matrix whose $i$-th row is $\left[1,\left(\mathbf{x}_{i}-\mathbf{x}\right)^{t}\right], \quad \mathbf{W}_{\mathbf{x}}=$ $\operatorname{diag}\left[K_{\mathbf{H}}\left(\mathbf{x}_{1}-\mathbf{x}\right), \ldots, K_{\mathbf{H}}\left(\mathbf{x}_{n}-\mathbf{x}\right)\right]$, with $K_{\mathbf{H}}(\mathbf{u})=|\mathbf{H}|^{-1} K\left(\mathbf{H}^{-1} \mathbf{u}\right)$, being $K$ a multivariate kernel function, and $\mathbf{H}$ is a $d \times d$ nonsingular symmetric matrix called the bandwidth matrix or smoothing matrix.

Note that, fixing a bandwidth matrix, $\hat{\mu}_{\mathbf{H}}(\mathbf{x})$ is a linear estimator, being $s_{\mathbf{x}}$ the so-called smoother vector. The estimated values at the sample locations are given by $\hat{\boldsymbol{\mu}}=\left[\hat{\mu}_{\mathbf{H}}\left(\mathbf{x}_{1}\right), \ldots, \hat{\mu}_{\mathbf{H}}\left(\mathbf{x}_{n}\right)\right]^{t}=\mathbf{S Y}$, being $\mathbf{S}$ the smoothing matrix whose $i$-th row is equal to $s_{\mathbf{x}_{i}}^{t}$ (the smoother vector for $\mathbf{x}=\mathbf{x}_{i}$ ).

Although the choice of the kernel function is of secondary importance, the bandwidth matrix plays an important role in the performance of the local linear estimator (3), since it controls the shape and the size of the local neighborhood used for estimating the spatial trend, which directly impacts the smoothness of the estimator. Therefore, in this case as in any other kernel-based estimator, in practice, data-driven bandwidth selection methods are needed. Different proposals have been made over the last decades, especially for independent data. These approaches include, among others, cross-validation methods, plugin selectors or bootstrap techniques (see, e.g. Fan and Gijbels, 1996). However, when the data are correlated, most of currently available data-driven smoothing parameter selection methods for nonparametric regression will often fail to provide useful results (Opsomer et al, 2001; Liu, 2001). Usually, some adjustments to the methods for independent data are performed in order to develop criteria that provide reliable bandwidths when the data are correlated.

When spatial data are correlated, as it is assumed in the present paper, to select the bandwidth matrix for (3), we recommend the use of the "bias corrected and estimated" generalized cross-validation (CGCV) criterion, proposed in Francisco-Fernández and Opsomer (2005). This method is based on the generalized cross-validation (GCV) criterion (Craven and Wahba, 1978), suitably adjusted for the presence of spatial correlation. It consists in selecting 
the smoothing parameter $\mathbf{H}$ that minimizes:

$$
\operatorname{CGCV}(\mathbf{H})=\frac{1}{n} \sum_{i=1}^{n}\left[\frac{Y\left(\mathbf{x}_{i}\right)-\hat{\mu}_{\mathbf{H}}\left(\mathbf{x}_{i}\right)}{1-\frac{1}{n} \operatorname{tr}(\mathbf{S} \hat{\mathbf{R}})}\right]^{2},
$$

where for any square matrix $\mathbf{A}, \operatorname{tr}(\mathbf{A})$ stands for its trace, and $\hat{\mathbf{R}}$ is an estimate of the correlation matrix of the observations (incorporating the spatial dependence structure of the data). While Francisco-Fernández and Opsomer (2005) used the method of the moments to obtain this estimate, we employ a nonparametric estimator of the covariogram function $C(\cdot)$. The matrix $\hat{\mathbf{R}}$ can be obtained using this estimate as explained below.

Apart from the trend $\mu(\cdot)$, another key element to be estimated in model (2) is the spatial dependence of the process, modeled by the covariagram function $C(\cdot)$. However, in practice in geostatistics, it is preferred to estimate the spatial dependence through the semivariogram $\gamma(\mathbf{u})=C(\mathbf{0})-C(\mathbf{u})$.

Similarly to the trend, a local linear estimation of the variogram $\hat{\gamma}(\mathbf{u})$ can be obtained from the residuals $\mathbf{r}=\left(r_{1}, \ldots, r_{n}\right)^{t}=\mathbf{Y}-\mathbf{S Y}$, applying (3) to

$$
\left\{\left(\mathbf{x}_{i}-\mathbf{x}_{j}, \frac{1}{2}\left(r_{i}-r_{j}\right)^{2}\right): 1 \leq i<j \leq n\right\} .
$$

However, it is well-known that the direct use of the residuals gives rise to biased variogram estimates, underestimating the variability of the spatial process (see, e.g. Cressie, 1993, Section 3.4.3). Denoting by $\boldsymbol{\Sigma}$ the covariance matrix of the errors, the covariance of the residuals is:

$$
\operatorname{Var}(\mathbf{r})=\boldsymbol{\Sigma}+\mathbf{S} \boldsymbol{\Sigma} \mathbf{S}^{t}-\mathbf{\Sigma} \mathbf{S}^{t}-\mathbf{S} \boldsymbol{\Sigma}=\boldsymbol{\Sigma}_{\mathbf{r}}
$$

Therefore, $\mathbf{S} \boldsymbol{\Sigma} \mathbf{S}^{t}-\boldsymbol{\Sigma} \mathbf{S}^{t}-\mathbf{S} \boldsymbol{\Sigma}$ is a square matrix representing the bias derived by the direct use of the residuals. As it may have a significant impact on risk assessment, a similar approach to that described in Fernández-Casal and Francisco-Fernández (2014) is used to obtain approximately unbiased variogram estimates. First, applying a local linear fit, a residual semivariogram $\tilde{\gamma}_{\mathbf{r}}(\cdot)$ is computed directly from $\mathbf{r}$. Then, using an iterative algorithm, the squared differences of the residuals are conveniently corrected and used to compute a pilot local linear variogram estimate $\tilde{\gamma}(\cdot)$. Then, valid variogram estimates are obtained by fitting "nonparametric" isotropic Shapiro-Botha models (Shapiro and Botha, 1991) to the nonparametric pilot estimates (see Fernández-Casal et al, 2018, for a detailed description of the algorithm), which will be denoted by $\hat{\gamma}_{\mathbf{r}}(\cdot)$ and $\hat{\gamma}(\cdot)$, respectively.

As described above, the estimation of the variogram is performed from the residuals, so it is necessary to previously estimate the trend. Nevertheless, as it was also discussed, to efficiently estimate the trend, the bandwidth should be properly selected by taking the spatial dependence into account, so this 
would be a circular problem. This problem can be solved using the iterative approach proposed in Fernández-Casal and Francisco-Fernández (2014). At each iteration, the CGCV criterion (4), jointly with the approximation of the variogram obtained in the previous iteration, is used to select the bandwidth to estimate the trend (the initial bandwidth could be selected assuming independence). Then, the trend is estimated and the corresponding residuals are used to update the variogram estimate. This approach is implemented in the np.fitgeo function of the npsp package (Fernandez-Casal, 2021).

\section{Conditional bootstrap algorithm}

In this section, the bootstrap algorithm to estimate the conditional risk is presented. This method is based on a general conditional simulation method combining unconditional simulations with kriging predictions (see, e.g. Chilès and Delfiner, 2012, Section 7.3.1). In a first step, the bootstrap algorithm studied in Fernández-Casal et al (2018) conveniently modified is used to generate the unconditional replicas. These authors proposed a bootstrap procedure to estimate the unconditional risk by using Cholesky's decompositions of covariance matrices. In their approach, the estimated unconditional probability at a non-observed location $\mathbf{x}_{\alpha}^{e}$ is obtained from kriging predictions, whose values in practice smooth the true spatial fluctuation of the data (see, e.g. Journel, 1974). As this could affect the estimation of the exceedance probability, we propose the following algorithm to generate unconditional bootstrap replicas $Y_{N S}^{*}\left(\mathbf{x}_{\alpha}^{e}\right)$ at the estimation locations $\left\{\mathbf{x}_{\alpha}^{e}\right\}_{\alpha=1}^{n_{0}}$, in such a way that the estimated variability of the process is adequately reproduced:

1. Using the procedures described in Section 2.1,

(a) Compute $\hat{\mu}_{\mathbf{H}}(\cdot)$ at the observed locations and the corresponding residuals $\mathbf{r}$ to obtain $\hat{\gamma}_{\mathbf{r}}(\cdot)$ and its corrected version $\hat{\gamma}(\cdot)$.

(b) Form $\hat{\boldsymbol{\Sigma}}_{\mathbf{r}}$ from $\hat{\gamma}_{\mathbf{r}}(\cdot)$ and find the matrix $\mathbf{L}_{\mathbf{r}}$ such that $\hat{\boldsymbol{\Sigma}}_{\mathbf{r}}=\mathbf{L}_{\mathbf{r}} \mathbf{L}_{\mathbf{r}}^{t}$, using Cholesky decomposition.

(c) Form $\hat{\boldsymbol{\Sigma}}_{\alpha}$ corresponding to the estimation locations $\mathbf{x}_{\alpha}^{e}$ using $\hat{\gamma}(\cdot)$ and compute $\mathbf{L}_{\alpha}$ such that $\hat{\boldsymbol{\Sigma}}_{\alpha}=\mathbf{L}_{\alpha} \mathbf{L}_{\alpha}^{t}$.

(d) Compute the "uncorrelated" residuals $\mathbf{e}=\mathbf{L}_{\mathbf{r}}^{-1} \mathbf{r}$ and center them.

2. Generate a bootstrap sample as follows:

(a) Obtain independent bootstrap samples of size $n_{0}$ from $\mathbf{e}$, denoted by $\mathbf{e}^{*}$.

(b) Compute the unconditional bootstrap errors $\varepsilon_{N S}^{*}=\mathbf{L}_{\alpha} \mathbf{e}^{*}$.

(c) Obtain the unconditional bootstrap replicas

$$
Y_{N S}^{*}\left(\mathbf{x}_{\alpha}^{e}\right)=\hat{\mu}_{\mathbf{H}}\left(\mathbf{x}_{\alpha}^{e}\right)+\varepsilon_{N S}^{*}\left(\mathbf{x}_{\alpha}^{e}\right), \alpha=1, \ldots, n_{0} .
$$

Note that repeating step 2 a large number of times $B$, a bootstrap sample $Y_{N S}^{*(1)}\left(\mathbf{x}_{\alpha}^{e}\right), \ldots, Y_{N S}^{*(B)}\left(\mathbf{x}_{\alpha}^{e}\right)$ can be obtained, and the unconditional probability 
$r_{c}\left(\mathbf{x}_{\alpha}^{e}\right)=P\left[Y\left(\mathbf{x}_{\alpha}^{e}\right) \geq c\right]$ can be estimated by:

$$
\hat{r}_{c}\left(\mathbf{x}_{\alpha}^{e}\right)=\frac{1}{B} \sum_{j=1}^{B} I_{\left\{Y_{N S}^{*(j)}\left(\mathbf{x}_{\alpha}^{e}\right) \geq c\right\}},
$$

where $I_{\{\cdot\}}$ denotes the indicator function.

The proposed algorithm could be adapted for the stationary case, i.e. $\mu(\mathbf{x})=\mu$. Under this assumption, the variogram estimates do not need to be corrected and both covariance matrices, corresponding to the observed and the estimation locations, would be obtained directly from $\hat{\gamma}(\cdot)$. So, if $\hat{\boldsymbol{\Sigma}}=\mathbf{L L}^{t}$ and $\hat{\mu}$ is an estimate of the constant trend (for instance, the sample mean of the observed values), then,

$$
Y_{N S}^{*}\left(\mathbf{x}_{\alpha}^{e}\right)=\hat{\mu}+\varepsilon_{N S}^{*}\left(\mathbf{x}_{\alpha}^{e}\right),
$$

where $\varepsilon_{N S}^{*}=\mathbf{L}_{\alpha} \mathbf{e}^{*}$ and the bootstrap resample $\mathbf{e}^{*}$ is obtained from $\mathbf{e}=$ $\mathbf{L}^{-1}(\mathbf{Y}-\hat{\mu})$.

The previous algorithm produces bootstrap replicas that have their mean and variance-covariance matrix equal to the corresponding estimates of the spatial process $Y(\cdot)$ (see, e.g. Cressie, 1993, Section 3.6.1). However, as these replicas does not necessarily coincide with the observed values at the sample locations, this algorithm should not be used for the estimation of the conditional risk and it should be suitably modified (see, e.g. Chilès and Delfiner, 2012, Chapter 7).

Next the proposed bootstrap algorithm to estimate the conditional risk (1) is described. As a starting point, let us assume that, in (2), the trend is known and the true errors $\varepsilon=\left[\varepsilon\left(\mathbf{x}_{1}\right), \ldots, \varepsilon\left(\mathbf{x}_{n}\right)\right]^{t}$ are observed. The principle of the conditional simulation of the error at a location $\mathbf{x}_{\alpha}$ (see, e.g. Journel, 1974) is based on the trivial decomposition

$$
\varepsilon\left(\mathbf{x}_{\alpha}^{e}\right)=\hat{\varepsilon}\left(\mathbf{x}_{\alpha}^{e}\right)+\left[\varepsilon\left(\mathbf{x}_{\alpha}^{e}\right)-\hat{\varepsilon}\left(\mathbf{x}_{\alpha}^{e}\right)\right],
$$

where $\hat{\varepsilon}\left(\mathbf{x}_{\alpha}^{e}\right)$ is the simple kriging prediction at $\mathbf{x}_{\alpha}^{e}$ computed from $\varepsilon$. The idea is to substitute the unknown kriging error (the second term on the right hand side of (5)) by a simulation of this error, obtained from an unconditional simulation $\varepsilon_{N S}(\mathbf{x})$ of the process. Then, a conditional simulation of the error process is:

$$
\varepsilon_{C S}\left(\mathbf{x}_{\alpha}\right)=\hat{\varepsilon}\left(\mathbf{x}_{\alpha}\right)+\left[\varepsilon_{N S}\left(\mathbf{x}_{\alpha}\right)-\hat{\varepsilon}_{N S}\left(\mathbf{x}_{\alpha}\right)\right] .
$$

where $\hat{\varepsilon}_{N S}\left(\mathbf{x}_{\alpha}\right)$ is the kriging prediction at $\mathbf{x}_{\alpha}$ obtained from the unconditional simulations $\varepsilon_{N S}\left(\mathbf{x}_{i}\right), i=1, \ldots, n$, at the sample locations. Proceeding in this way, it is easy to verify that $\varepsilon_{C S}\left(\mathbf{x}_{i}\right)=\varepsilon\left(\mathbf{x}_{i}\right)$ and, in the case of simple kriging, $\operatorname{Cov}\left[\varepsilon_{C S}(\mathbf{x}], \varepsilon_{C S}(\mathbf{x}+\mathbf{u})\right]=C(\mathbf{u})$ (see, e.g. Chilès and Delfiner, 2012, Section 7.3.1). These properties guarantee that the simulations reproduce the behavior of the observed data, keeping the dependence structure of the spatial process. 
Taking into account the previous results and that the theoretical trends of the bootstrap replicates are the trend estimates, the proposed bootstrap algorithm to estimate the conditional risk follows these steps:

1. Use the unconditional bootstrap algorithm previously described to (jointly) generate $\varepsilon_{N S}^{*}\left(\mathbf{x}_{\alpha}^{e}\right), \alpha=1, \ldots, n_{0}$, and $\varepsilon_{N S}^{*}\left(\mathbf{x}_{i}\right), i=1, \ldots, n$.

2. Compute the simple kriging predictions $\hat{\varepsilon}\left(\mathbf{x}_{\alpha}^{e}\right)$ and $\hat{\varepsilon}_{N S}^{*}\left(\mathbf{x}_{\alpha}^{e}\right)$ from the observed residuals $\mathbf{r}$ and from the bootstrap errors $\varepsilon_{N S}^{*}\left(\mathbf{x}_{i}\right)$, respectively.

3. Considering equation (6), obtain the conditional bootstrap errors

$$
\varepsilon_{C S}^{*}\left(\mathbf{x}_{\alpha}^{e}\right)=\hat{\varepsilon}\left(\mathbf{x}_{\alpha}^{e}\right)+\left[\varepsilon_{N S}^{*}\left(\mathbf{x}_{\alpha}^{e}\right)-\hat{\varepsilon}_{N S}^{*}\left(\mathbf{x}_{\alpha}^{e}\right)\right]
$$

4. Compute the conditional bootstrap replicates

$$
Y_{C S}^{*}\left(\mathbf{x}_{\alpha}^{e}\right)=\hat{\mu}_{\mathbf{H}}\left(\mathbf{x}_{\alpha}^{e}\right)+\varepsilon_{C S}^{*}\left(\mathbf{x}_{\alpha}^{e}\right) .
$$

5. Repeat steps 1 to 4 a large number of times $B$ to obtain

$$
Y_{C S}^{*(1)}\left(\mathbf{x}_{\alpha}^{e}\right), \ldots, Y_{C S}^{*(B)}\left(\mathbf{x}_{\alpha}^{e}\right)
$$

6. Compute

$$
\hat{r}_{c}\left(\mathbf{x}_{\alpha}^{e}, \mathbf{Y}\right)=\frac{1}{B} \sum_{j=1}^{B} I_{\left\{Y_{C S}^{*(j)}\left(\mathbf{x}_{\alpha}^{e}\right) \geq c\right\}}
$$

as the approximation of (1).

\section{Simulation studies}

In this section, the conditional bootstrap procedure described in the previous section is numerically analyzed considering different scenarios. Regular grids in the unit square of sizes $n_{1}=15 \times 15,20 \times 20$ and $30 \times 30$ were considered. The top right diagonal sites were set as the estimation locations $\mathbf{x}_{\alpha}^{e}, \alpha=1, \ldots, n_{0}$, and the remaining ones as the observation sample $\mathbf{x}_{i}, i=1, \ldots, n$ (note that $\left.n=n_{1}-n_{0}\right)$. For example, the left panel of Figure 1 shows the estimation (triangles) and observation (circles) locations for the case of $n_{1}=20 \times 20$.

In the first part of the study, a nonstationary scenario was considered, generating $N=1000$ samples following model (2) on the sample locations, with mean function $\mu\left(x_{1}, x_{2}\right)=2.5+\sin \left(2 \pi x_{1}\right)+4\left(x_{2}-0.5\right)^{2}$ (see the right panel of Figure 1). The random errors in (2) were simulated as normally distributed with zero mean and isotropic exponential covariogram,

$$
\gamma_{\theta}(\mathbf{u})=c_{0}+c_{1}\{1-\exp [-3\|\mathbf{u}\| / a]\}
$$

(for $\mathbf{u} \neq \mathbf{0}$ ), where $c_{0}$ is the nugget effect, $c_{1}$ is the partial sill $\left(c_{1}=1-c_{0}\right)$ and $a$ is the practical range. The values considered were: $a=0.3,0.6$ and 0.9 , $c_{0}=0,0.2,0.4$ and 0.8 . 
(a)

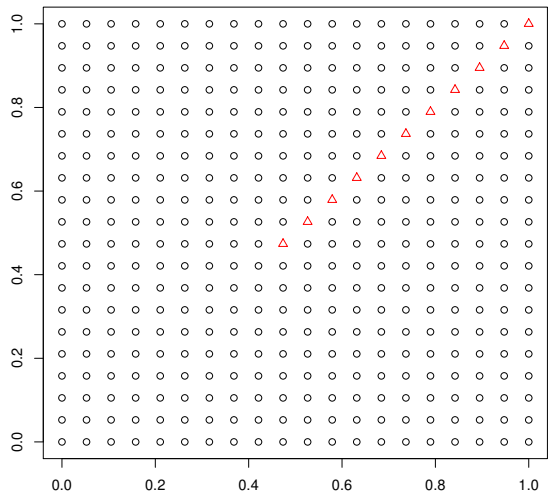

(b)

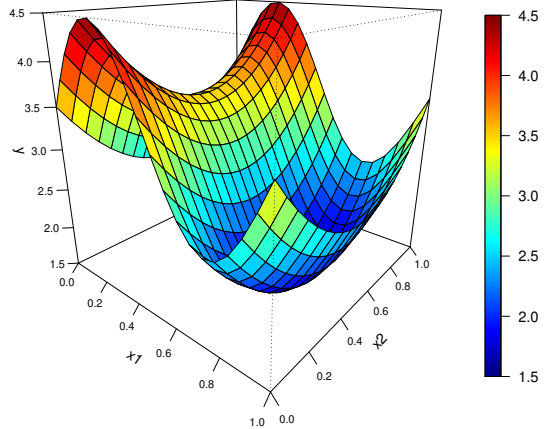

Fig. 1 (a) Sample and estimation locations (circles and triangles, respectively) for $n_{1}=$ $20 \times 20$ and (b) theoretical trend.

To apply the conditional bootstrap algorithm described in Section 3, firstly, it is necessary to estimate the trend $\mu(\cdot)$. For this, we employed the local linear estimator (3), with a multiplicative triweight kernel. To reduce the computing time and to avoid the effect of the bandwidth selection criterion used for trend estimation in the results, the bandwidth $\mathbf{H}_{\mathrm{MASE}}$ minimizing the mean average squared error,

$$
\operatorname{MASE}(\mathbf{H})=\frac{1}{n}(\mathbf{S} \boldsymbol{\mu}-\boldsymbol{\mu})^{t}(\mathbf{S} \boldsymbol{\mu}-\boldsymbol{\mu})+\frac{1}{n} \operatorname{tr}\left(\mathbf{S} \boldsymbol{\Sigma} \mathbf{S}^{t}\right),
$$

where $\boldsymbol{\mu}=\left[\mu\left(\mathbf{x}_{\mathbf{1}}\right), \ldots, \mu\left(\mathbf{x}_{\mathbf{n}}\right)\right]^{t}$, was employed. This optimal bandwidth cannot be used in practice, since its computation depends on the unknown trend $\mu(\cdot)$. In that case, we recommend the use of the CGCV criterion (4) that provided very similar results to those obtained with $\mathbf{H}_{\mathrm{MASE}}$ in some simulation experiments (Fernández-Casal et al, 2018). As for the dependence estimation, applying the iterative algorithm described in the Section 2.1, the residual variogram $\hat{\gamma}_{\mathbf{r}}(\cdot)$ and its bias-corrected version $\hat{\gamma}(\cdot)$ were obtained.

As an example, Figure 2 shows the average of the nonparametric trend estimates (left panel) and the residual and bias-corrected variogram estimates (right panel) computed over the $N=1000$ simulated samples, for $n_{1}=20 \times 20$, $c_{0}=0.2$, and $a=0.6$. It can be observed that both, the local linear trend and the bias-corrected variogram estimates, provided good approximations to their theoretical versions.

At each simulation, using the trend and variogram estimates, the algorithm proposed in Section 3 was applied with $B=1000$ bootstrap replicas. For each $\alpha=1, \ldots, n_{0}$, the conditional probabilities $r_{c}\left(\mathbf{x}_{\alpha}^{e}, \mathbf{Y}\right)$ were estimated by $\hat{r}_{c}\left(\mathbf{x}_{\alpha}^{e}, \mathbf{Y}\right)$, given in (7), considering threshold values $c=2,3$ and 4 . At each estimation location $\mathbf{x}_{\alpha}^{e}, \alpha=1, \ldots, n_{0}$, the errors $\hat{r}_{c}\left(\mathbf{x}_{\alpha}^{e}, \mathbf{Y}\right)-r_{c}\left(\mathbf{x}_{\alpha}^{e}, \mathbf{Y}\right)$ and the squared errors $\left[\hat{r}_{c}\left(\mathbf{x}_{\alpha}^{e}, \mathbf{Y}\right)-r_{c}\left(\mathbf{x}_{\alpha}^{e}, \mathbf{Y}\right)\right]^{2}$ were computed to evaluate 
(a)

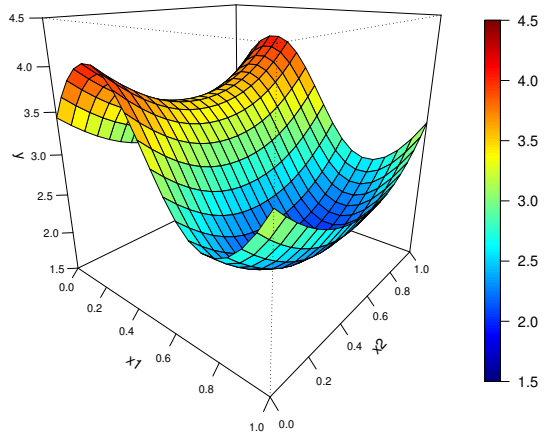

(b)

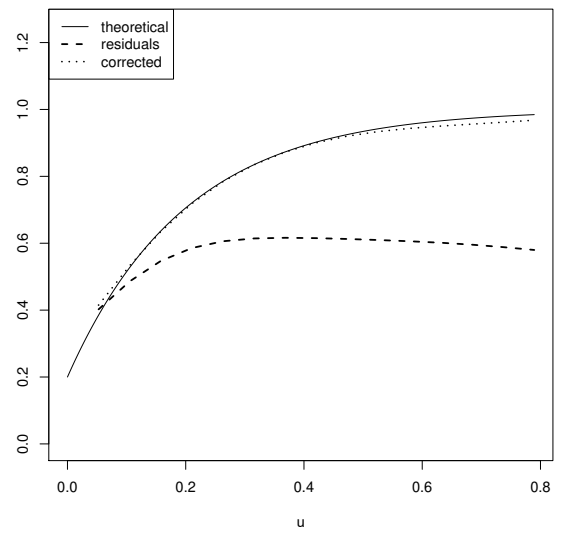

Fig. 2 (a) Averages of the local linear trend estimates, and (b) the residual and biascorrected variogram estimates (dashed and dotted lines respectively; the solid line represents the theoretical variogram) over the simulated samples, for $n_{1}=20 \times 20, c_{0}=0.2$, and $a=0.6$.

the performance of this procedure (named "corrected"). Note that, taking into account that the responses are normally distributed, the theoretical probabilities $r_{c}\left(\mathbf{x}_{\alpha}^{e}, \mathbf{Y}\right)$ can be obtained as:

$$
1-\Phi\left(\frac{c-\hat{Y}_{S K}\left(\mathbf{x}_{\alpha}^{e}\right)}{\hat{\sigma}_{S K}\left(\mathbf{x}_{\alpha}^{e}\right)}\right)
$$

being $\Phi$ the standard normal cumulative distribution function, $\hat{Y}_{S K}\left(\mathbf{x}_{\alpha}^{e}\right)$ the simple kriging prediction of $Y\left(\mathbf{x}_{\alpha}^{e}\right)$, obtained using the theoretical trend and covariance matrix, and $\hat{\sigma}_{S K}^{2}\left(\mathbf{x}_{\alpha}^{e}\right)$ the corresponding simple kriging variance.

For the sake of brevity, only some representative results are shown here. For example, Table 1 shows the mean, median and standard deviations of the squared errors $\left(\times 10^{-2}\right)$ of the estimates obtained with the "corrected" approach, for $a=0.6, c_{0}=0.2$, and the different threshold values and sample sizes considered. It can be observed that, for the different values of $c$, the mean squared error (MSE) decreases as the sample size $n$ increases, suggesting the possible consistency of the conditional probability estimator.

Table 1 Mean, median and standard deviations of the squared errors $\left(\times 10^{-2}\right)$ of the "corrected" procedure, for $a=0.6$ and $c_{0}=0.2$.

\begin{tabular}{c|ccc|ccc|ccc}
\hline & \multicolumn{3}{|c|}{$n_{1}=15 \times 15$} & \multicolumn{3}{c|}{$n_{1}=20 \times 20$} & \multicolumn{3}{c}{$n_{1}=30 \times 30$} \\
\hline$c$ & mean & median & sd & mean & median & sd & mean & median & sd \\
\hline 2 & 0.34 & 0.04 & 1.31 & 0.21 & 0.03 & 0.82 & 0.10 & 0.01 & 0.43 \\
3 & 0.31 & 0.03 & 1.02 & 0.19 & 0.01 & 0.74 & 0.09 & 0.01 & 0.35 \\
4 & 0.13 & 0.00 & 0.49 & 0.07 & 0.00 & 0.36 & 0.04 & 0.00 & 0.20 \\
\hline
\end{tabular}


The good performance of the "corrected" approach can also be observed in Figure 3. It contains boxplots of the theoretical (left panel) and estimated (right panel) conditional probabilities of exceeding a threshold of $c=3$ at the estimation locations $\mathbf{x}_{\alpha}^{e}$, using the "corrected" method and considering $n_{1}=15 \times 15, a=0.6$ and $c_{0}=0.2$. A very similar pattern of the corresponding boxplots for the theoretical and the estimated conditional risks at all estimation locations is observed in this figure.

(a)

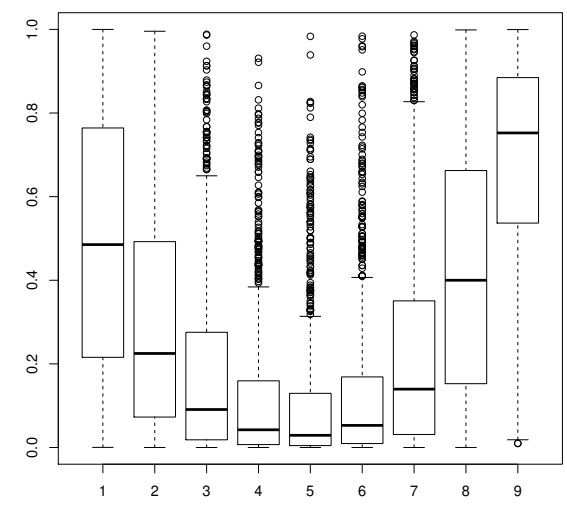

(b)

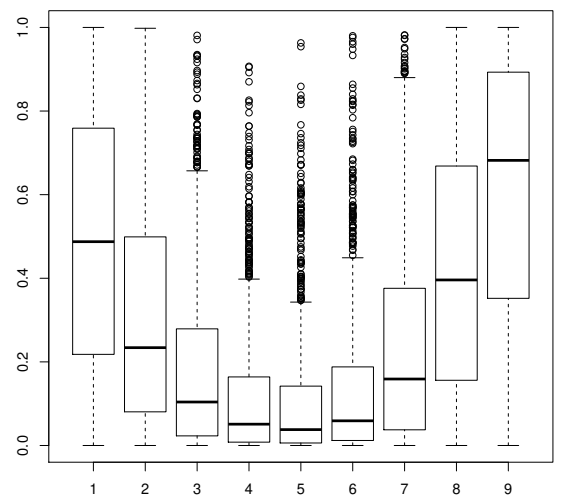

Fig. 3 Boxplots of the theoretical (a) and estimate (b) conditional probabilities of exceeding a threshold of $c=3$ using the "corrected" method, for $n_{1}=15 \times 15, a=0.6$ and $c_{0}=0.2$, at different estimation locations $\mathbf{x}_{\alpha}^{e}, \alpha=1, \ldots, 9$.

Completing the information in Figure 3, scatterplots of the theoretical versus the estimated conditional exceeding probabilities obtained from the $N=1000$ simulated samples, at the estimation locations $\mathbf{x}_{1}^{e}=(0.428,0.428)$ and $\mathbf{x}_{5}^{e}=(0.714,0.714)$ are shown in Figure 4 . It can be observed the closeness between the corresponding theoretical and the estimated probabilities computed in each simulated sample. In general, a similar good behavior of the proposed procedure was observed in all simulation settings.

Two additional approaches were used to estimate the conditional exceeding probabilities, the IK method, briefly mentioned in the Introduction, and a "biased" procedure similar to the one shown in Section 3, but using $\hat{\gamma}_{\mathbf{r}}(\cdot)$ instead of $\hat{\gamma}(\cdot)$ to generate the unconditional replicas $\varepsilon_{N S}^{*}(\cdot)$. In the IK method only the observed values of the indicator variable $I_{\left\{Y\left(\mathbf{x}_{0}\right) \geq c\right\}}$ are considered and, assuming that they are stationary, simple kriging is performed to compute predictions at the estimation locations, since

$$
P\left[Y\left(\mathbf{x}_{0}\right) \geq c \mid \mathbf{Y}\right]=\mathbb{E}\left[I_{\left\{Y\left(\mathbf{x}_{0}\right) \geq c\right\}} \mid \mathbf{Y}\right]
$$

(see, e.g. Chilès and Delfiner, 2012, Section 6.3.3, for further details). In practice, this parametric approach was implemented using the geo $\mathrm{R}$ package (Ribeiro Jr et al, 2020), fitting an exponential variogram model to the indicator 

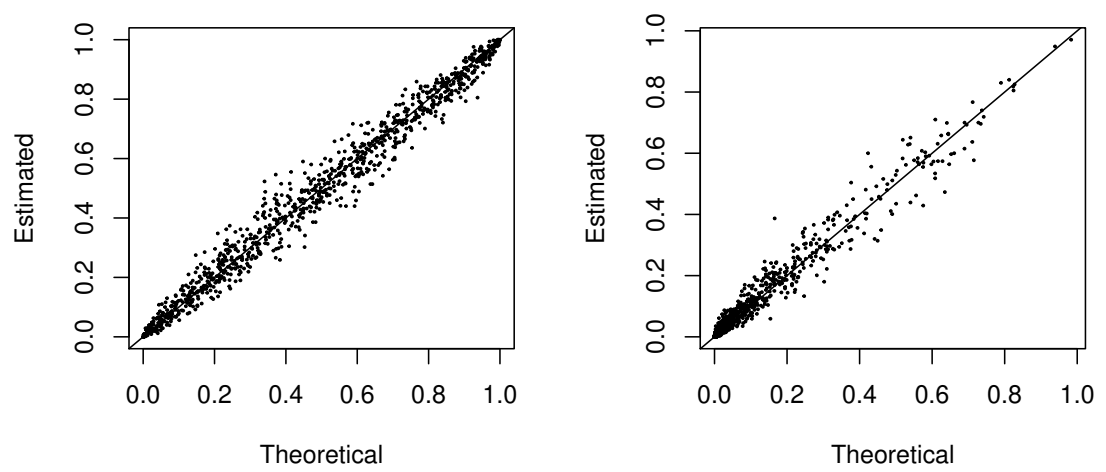

Fig. 4 Scatterplots of theoretical (x-axis) and estimated (y-axis) probabilities of exceeding a threshold of $c=3$ using the "corrected" method, for $n_{1}=15 \times 15, a=0.6$ and $c_{0}=0.2$, at the estimation locations $\mathbf{x}_{\alpha}^{e}$, for $\alpha=1$ (left) and $\alpha=5$ (right).

variable (assuming a constant trend). The performance of these two methods was compared with that obtained by the "corrected" approach in the previous scenarios.

As example, Table 2 compares the results obtained with the IK, the "biased" and the "corrected" approaches, considering the previous nonstationary process with $n_{1}=20 \times 20, a=0.3,0.6,0.9, c_{0}=0.2$, and the different threshold values. It can be observed that the IK method has the highest error values, which may be due to the non-stationarity of the process. The bias effect is also reflected in the MSE values of the "biased" approach, being higher than those obtained by the "corrected" technique. This makes clear the effectiveness of performing the bias-correction in the variogram estimation. In addition, all the MSE values tend to be smaller as the range $a$ increases, suggesting that the effect of the spatial dependence is similar in all cases.

Table 2 Mean and standard deviations of the squared errors $\left(\times 10^{-2}\right)$ of the IK, the "biased" and the "corrected" procedures, for $n_{1}=20 \times 20, a=0.3,0.6,0.9$ and $c_{0}=0.2$.

\begin{tabular}{c|c|cc|cc|cc}
\hline & & \multicolumn{2}{|c|}{ IK } & \multicolumn{2}{c|}{ Biased } & \multicolumn{2}{c}{ Corrected } \\
\hline$c$ & $a$ & mean & sd & mean & sd & mean & sd \\
\hline \multirow{3}{*}{2} & 0.3 & 1.26 & 2.05 & 0.38 & 1.03 & 0.31 & 0.95 \\
& 0.6 & 1.16 & 2.10 & 0.26 & 0.94 & 0.21 & 0.82 \\
& 0.9 & 1.11 & 2.22 & 0.22 & 0.85 & 0.17 & 0.74 \\
\hline \multirow{4}{*}{3} & 0.3 & 1.25 & 2.38 & 0.34 & 1.12 & 0.29 & 0.93 \\
& 0.6 & 1.08 & 2.26 & 0.24 & 0.95 & 0.19 & 0.74 \\
& 0.9 & 1.00 & 2.34 & 0.20 & 0.84 & 0.16 & 0.66 \\
\hline \multirow{4}{*}{4} & 0.3 & 0.65 & 1.96 & 0.14 & 0.64 & 0.12 & 0.51 \\
& 0.6 & 0.62 & 2.07 & 0.10 & 0.50 & 0.07 & 0.36 \\
& 0.9 & 0.58 & 2.07 & 0.08 & 0.44 & 0.06 & 0.30 \\
\hline
\end{tabular}

Similar conclusions can be deduced from Figure 5, where the boxplots of the estimation errors, $\hat{r}_{c}\left(\mathbf{x}_{\alpha}^{e}, \mathbf{Y}\right)-r_{c}\left(\mathbf{x}_{\alpha}^{e}, \mathbf{Y}\right)$, obtained in each estimation location 
$\mathbf{x}_{\alpha}^{e}$ by the three procedures, considering the same scenario $n_{1}=20 \times 20$, $a=0.6, c_{0}=0.2$ and a threshold of $c=3$ are shown. The behavior observed in this setting (illustrated in Table 2 and Figure 5) is similar to that obtained in the other simulated scenarios.

(a)

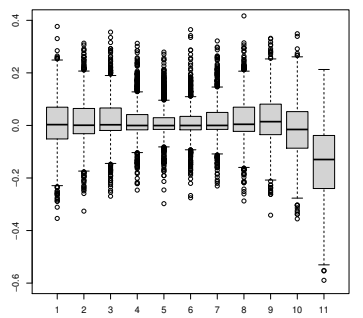

(b)

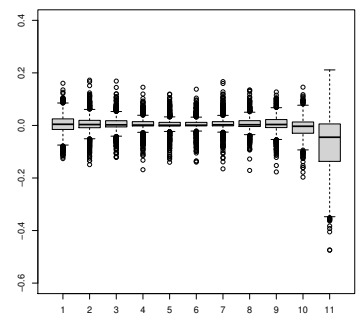

(c)

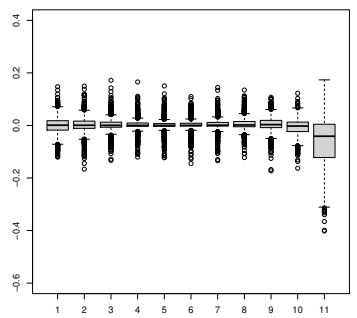

Fig. 5 Boxplots of estimation errors for (a) the IK, (b) the "biased" and (c) the "corrected" methods, for $n_{1}=20 \times 20, a=0.6, c_{0}=0.2$ and considering a threshold of $c=3$.

In the second part of the study, we repeat the same type of numerical experiments, but considering a stationary process. This avoids the trend estimation effect, giving some advantage to the IK procedure. Specifically, we consider model (2), with errors simulated as in the first part of the study, but the trend function was now fixed to $\mu(\mathbf{x})=3$. In this framework, the "biased" procedure was not applied, instead a so-called "constant" method was employed. In this procedure, the replicas were obtained using the modification of the unconditional bootstrap algorithm for stationary processes described in Section 3, where the covariance matrix $\boldsymbol{\Sigma}$ is derived directly from the variogram of $Y$. The "corrected" procedure was also used, assuming (wrongly) the presence of a non-constant trend.

As an example of the results obtained in this stationary setting, Table 3 shows the mean, the median and the standard deviations of the squared errors $\left(\times 10^{-2}\right)$ of the estimates obtained with the IK, the "corrected" and the "constant" approaches, for $n_{1}=20 \times 20, a=0.6$ and $c_{0}=0.2$. As observed, even with a constant trend, the IK method provided higher MSE values than those obtained by the other procedures. This seems to be another limitation of this method due to the loss of information by using only the discretized response values (see, e.g. Tolosana-Delgado et al, 2008). Unsurprisingly, the errors obtained with the "corrected" method are slightly higher than those achieved with the "constant" approach, although they are still much lower than the ones of the IK procedure. Therefore, even when a non-constant trend is incorrectly assumed, the "corrected" method is a relatively valid alternative for estimating the conditional probability. In fact, in our experiments, we have observed that the estimated probabilities are pretty similar when using the "corrected" and the "constant" methods, especially at interior points, as can be seen in Figure 6 . 
Table 3 Mean, median and standard deviations of the squared errors $\left(\times 10^{-2}\right)$ of the IK, the "corrected" and the "constant" procedures, for $n_{1}=20 \times 20, a=0.6$ and $c_{0}=0.2$, considering $\mu(\mathbf{x})=3$.

\begin{tabular}{c|ccc|ccc|ccc}
\hline & \multicolumn{3}{|c|}{ IK } & \multicolumn{3}{c|}{ Corrected } & \multicolumn{3}{c}{ Constant } \\
\hline$c$ & mean & median & sd & mean & median & sd & mean & median & sd \\
\hline 2 & 0.69 & 0.13 & 1.52 & 0.11 & 0.01 & 0.35 & 0.09 & 0.01 & 0.28 \\
3 & 1.07 & 0.42 & 1.64 & 0.18 & 0.04 & 0.46 & 0.17 & 0.04 & 0.36 \\
4 & 0.73 & 0.14 & 1.55 & 0.10 & 0.01 & 0.35 & 0.09 & 0.01 & 0.30 \\
\hline
\end{tabular}

(a)

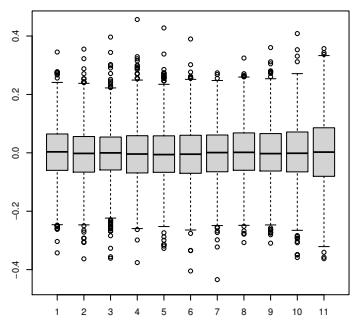

(b)

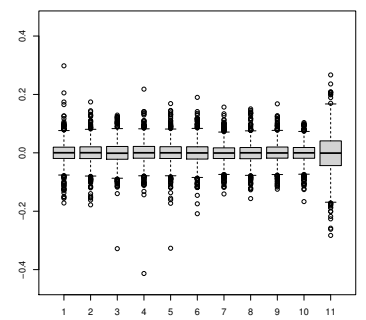

(c)

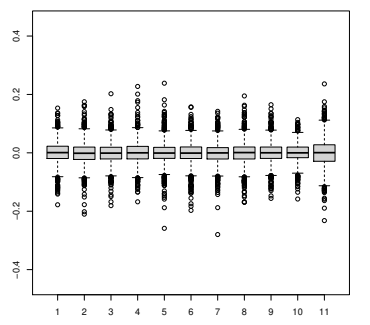

Fig. 6 Boxplots of estimation errors for (a) the IK, (b) the "corrected" and (c) the "constant" methods, for $n_{1}=20 \times 20, a=0.6, c_{0}=0.2$ and $c=3$, considering $\mu(\mathbf{x})=3$.

\section{Application to real data}

The proposed methodology was applied to a data set containing the total precipitations (square-root of rainfall inches) during March 2016 recorded over 1053 locations on the continental part of USA, included in Figure 7(a). This data set is supplied with the $\mathrm{R}$ package npsp. The trend and variogram estimates were obtained using the iterative algorithm described in Section 2.1, implemented in the function np.fitgeo of that package. The final trend estimate is shown in Figure 7(b) and Figure 7(c) shows the residual variogram $\hat{\gamma}_{\mathbf{r}}(\cdot)$ (dashed line) and the bias-corrected estimate $\hat{\gamma}(\cdot)$ (solid line). Using these estimates, the kriging predictions were computed (Figure $7(\mathrm{~d})$ ).

Estimated probability maps were computed for different threshold values (square-root of rainfall inches), $c=\{1.0,1.5,2.0,2.5,3.0,3.5,4.0\}$, by applying the two bootstrap algorithms (unconditional and conditional) described in Section 3 with $B=1000$ bootstrap replicas. For reason of space, only the case of threshold $c=2.0$ is included here (Figure 8).

In Figure 8, it can be observed that the unconditional bootstrap provides smoother estimates than those obtained with the conditional approach, emphasizing the dominant effect of the trend estimate (large-scale variability). On the other hand, the conditional map shows higher variability, reaching the extreme values 0 and 1 at sample locations. These differences are more evident in the northern regions of the central area of the map, where the proposed method produces estimates in line with the observed values (shown in Figure 7(a)), due to the stronger effect of the spatial dependence (small-scale variability) on the conditional estimates. 
(a)

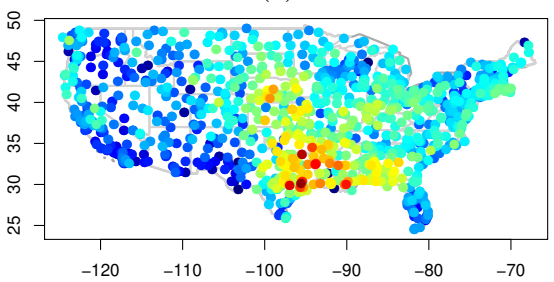

(c)

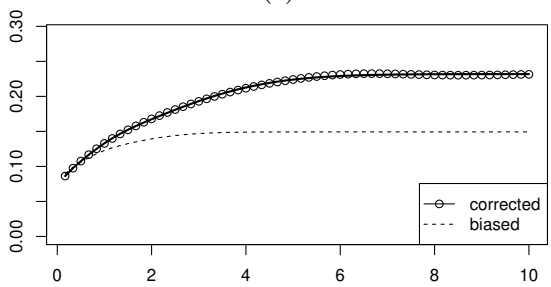

(b)

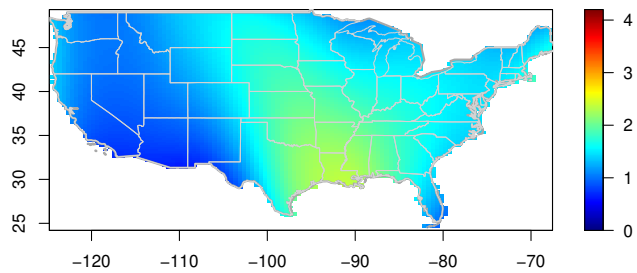

(d)

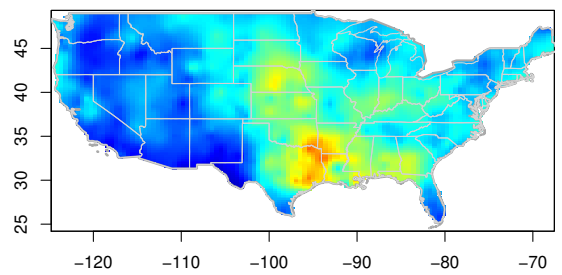

Fig. 7 (a) Spatial locations and observed values, (b) nonparametric trend estimates, (c) semivariogram estimates, and (d) kriging predictions

(a)

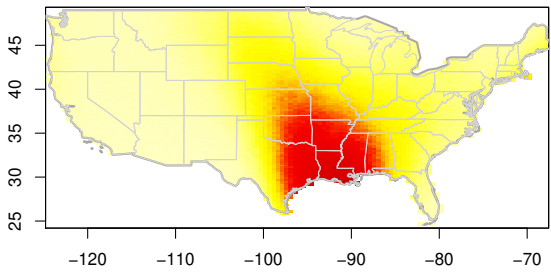

(b)

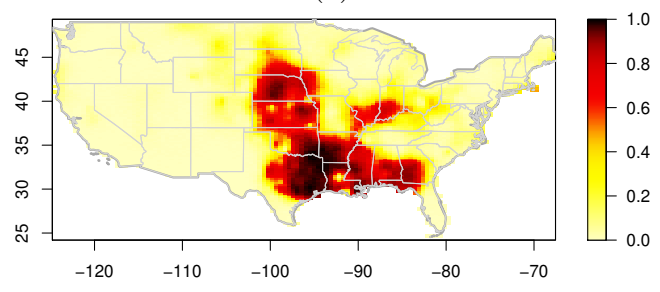

Fig. 8 Estimated unconditional (a) and conditional (b) risk maps for $c=2.0$.

\section{Discussion and further remarks}

A bootstrap algorithm to estimate threshold exceeding conditional probabilities is proposed and numerically analyzed in this paper. The probabilities are approximated from bootstrap conditional replicates obtained in a two-stage procedure. In the first step, a modification of the unconditional bootstrap method proposed in Fernández-Casal et al (2018) is used, in such a way that the variability of the process is better reproduced at prediction locations. In the second step, the unconditional replicates are combined with kriging predictions so that the resulting values coincide with the observed values at the sample locations.

Unlike traditional methods, such as IK or DK, the new approach is designed to be applied for processes that are not stationary in the mean. Although it can be conveniently adapted to the case of considering a constant mean, in the simulations performed, we have observed that the proposed ("corrected") approach provided much better results that the IK method even for stationary processes. 
Moreover, the new approach is fully nonparametric and, therefore, problems due to model misspecification are at least partially avoided. The trend is estimated using the local linear estimator, although other linear smoothers could also be used. Additionally, a "bias-corrected" method to estimate the spatial dependence is employed. In this way, the proposed bootstrap algorithm takes into account that the variability of the residuals is not equal to that of the true errors. In the results of the simulations, it is observed the benefits of correcting the bias caused by the direct use of residuals in variogram estimation, leading to more accurate conditional probability estimates.

The complete simulation study shows a good behavior of the new method and its better performance with respect to the IK technique in different scenarios, considering several degrees of spatial dependence and spatial trends. Although only regular sampling is included in this paper, we have also performed some simulations considering non-regular sampling designs. The results obtained follow the same line as those with regular sampling and similar conclusions could be deduced from them. Therefore, for the sake of brevity, we have preferred not to include then in the paper. Note that the case of nonregular design is also illustrated in the real data application in Section 5. It is important to remark that in the case of non-regular designs the computational cost is larger. In the fixed design case, the optimal bandwidth matrices (for trend and variogram estimation) and the smoothing matrix $\mathbf{S}$ only need to be computed once, whereas a random design will require the computation of these matrices at each iteration, considerably increasing the computational time.

The numerical analysis carried out in this research was performed with the statistical environment R ( R Development Core Team, 2022), using the functions for nonparametric trend and variogram estimation supplied with the npsp package (Fernandez-Casal, 2021).

\section{References}

Chilès J, Delfiner P (2012) Geostatistics: modeling spatial uncertainty, 2nd edn. Wiley, New York

Craven P, Wahba G (1978) Smoothing noisy data with spline functions. Numerische Mathematik 31(4):377-403

Cressie N (1993) Statistics for spatial data. Wiley, New York

Fan J, Gijbels I (1996) Local polynomial modelling and its applications. Chapman \& Hall, London

Fernandez-Casal R (2021) npsp: Nonparametric spatial (geo)statistics. R package version 0.7-8, https://rubenfcasal.github.io/npsp

Fernández-Casal R, Francisco-Fernández M (2014) Nonparametric biascorrected variogram estimation under non-constant trend. Stoch Environ 
Res Risk Assess 28(5):1247-1259

Fernández-Casal R, Castillo-Páez S, Francisco-Fernández M (2018) Nonparametric geostatistical risk mapping. Stoch Environ Res Risk Assess $32(3): 675-684$

Francisco-Fernández M, Opsomer JD (2005) Smoothing parameter selection methods for nonparametric regression with spatially correlated errors. Can J Stat 33:539-558

Goovaerts P, Webster R, Dubois JP (1997) Assessing the risk of soil contamination in the swiss jura using indicator geostatistics. Environ Ecol Stat $4(1): 49-64$

Journel AG (1974) Geostatistics for conditional simulation of ore bodies. Econ Geol 69(5):673-687

Lark R, Ferguson R (2004) Mapping risk of soil nutrient deficiency or excess by disjunctive and indicator kriging. Geoderma 118:39-53

Liu X (2001) Kernel smoothing for spatially correlated data. PhD thesis, Department of Statistics, Iowa State University

Opsomer JD, Wang Y, Yang Y (2001) Nonparametric regression with correlated errors. Stat Sci 16:134-153

R Development Core Team (2022) R: A Language and Environment for Statistical Computing. R Foundation for Statistical Computing, Vienna, Austria, http://www.R-project.org

Ribeiro Jr PJ, Diggle PJ, Schlather M, et al (2020) geoR: Analysis of Geostatistical Data. R package version 1.8-1, https://CRAN.R-project.org/package= geoR

Shapiro A, Botha JD (1991) Variogram fitting with a general class of conditionally nonnegative definite functions. Comput Stat Data Anal 11(1):87-96

Tolosana-Delgado R, Pawlowsky-Glahn V, Egozcue JJ (2008) Indicator kriging without order relation violations. Math Geosci 40(3):327-347

Wand MP, Jones MC (1995) Kernel smoothing. Chapman and Hall, London

Webster R, Oliver M (1989) Optimal interpolation and isarithmic mapping of soil properties. VI. Disjunctive kriging and mapping the conditional probability. J Soil Sci 40(3):497-512 


\section{Statements \& Declarations}

Funding The research of Rubén Fernández-Casal and Mario Francisco-Fernández has been supported by MINECO (Grant MTM2017-82724-R) and MICINN (Grant PID2020-113578RB-I00), and by the Xunta de Galicia (Grupos de Referencia Competitiva ED431C-2020-14 and Centro de Investigación del Sistema Universitario de Galicia ED431G 2019/01), all of them through the ERDF. The research of Sergio Castillo Páez has been supported by the Universidad de las Fuerzas Armadas ESPE, from Ecuador.

Competing Interests The authors have no relevant financial or non-financial interests to disclose.

Author Contributions All authors contributed equally to this work. 